\title{
Subthalamic Nucleus Local Field Potential Activity during the Eriksen Flanker Task Reveals a Novel Role for Theta Phase during Conflict Monitoring
}

\author{
Baltazar Zavala, ${ }^{1,2}$ John-Stuart Brittain, ${ }^{1}$ Ned Jenkinson, ${ }^{1}$ Keyoumars Ashkan, ${ }^{3}$ Thomas Foltynie, ${ }^{4}$ Patricia Limousin, ${ }^{4}$ \\ Ludvic Zrinzo, ${ }^{4}$ Alexander L. Green, ${ }^{1}$ Tipu Aziz, ${ }^{1}$ Kareem Zaghloul, ${ }^{2}$ and Peter Brown ${ }^{1}$ \\ ${ }^{1}$ Functional Neurosurgery-Experimental Neurology Group, Nuffield Department of Clinical Neurology, University of Oxford John Radcliffe Hospital, Oxford \\ OX3 9DU, United Kingdom, ${ }^{2}$ Surgical Neurology Branch, National Institutes of Health, Bethesda, Maryland 20814, ${ }^{3}$ Department of Neurosurgery, King's \\ College Hospital, Kings College, London SE5 9RS, United Kingdom, and ${ }^{4}$ Sobell Department of Motor Neuroscience and Movement Disorders, UCL \\ Institute of Neurology, London WC1 3BG, United Kingdom
}

The subthalamic nucleus (STN) is thought to play a central role in modulating responses during conflict. Computational models have suggested that the location of the STN in the basal ganglia, as well as its numerous connections to conflict-related cortical structures, allows it to be ideally situated to act as a global inhibitor during conflict. Additionally, recent behavioral experiments have shown that deep brain stimulation to the STN results in impulsivity during high-conflict situations. However, the precise mechanisms that mediate the "hold-your-horses" function of the STN remain unclear. We recorded from deep brain stimulation electrodes implanted bilaterally in the STN of 13 human subjects with Parkinson's disease while they performed a flanker task. The incongruent trials with the shortest reaction times showed no behavioral or electrophysiological differences from congruent trials, suggesting that the distracter stimuli were successfully ignored. In these trials, cue-locked STN theta band activity demonstrated phase alignment across trials and was followed by a periresponse increase in theta power. In contrast, incongruent trials with longer reaction times demonstrated a relative reduction in theta phase alignment followed by higher theta power. Theta phase alignment negatively correlated with subject reaction time, and theta power positively correlated with trial reaction time. Thus, when conflicting stimuli are not properly ignored, disruption of STN theta phase alignment may help operationalize the hold-your-horses role of the nucleus, whereas later increases in the amplitude of theta oscillations may help overcome this function.

\section{Introduction}

The role of the subthalamic nucleus (STN) during conflict has recently attracted increased attention (Brittain et al., 2012; Zaghloul et al., 2012). The STN, which is the most common target for deep brain stimulation (DBS) for the treatment of Parkinson's disease (PD), is traditionally postulated to play a role in disfacilitating movement (Albin et al., 1989; DeLong, 1990). Accordingly, it has been proposed that the STN may adjust the "evidence threshold" in a drift diffusion model of decision mak-

\footnotetext{
Received March 7, 2013; revised Aug. 3, 2013; accepted Aug. 9, 2013.

Author contributions:B.Z., J.-S.B., N.J., K.Z., and P.B. designed research; B.Z., J.-S.B., and N.J. performed research; B.Z., J.-S.B., K.Z., and P.B. analyzed data; B.Z., J.-S.B., N.J., K.A., T.F., P.L., L.Z., A.L.G., T.A., K.Z., and P.B. wrote the paper.

B.Z. is supported by the Rhodes Trust and the National Institutes of Health Oxford-Cambridge fellowship. T.F. P.L., and L.Z. are funded by the Department of Health National Institute for Health Research UCL Biomedical Research Center, The Monument Trust, and Parkinson's Appeal for Deep Brain Stimulation. P.B. is funded by the Medical Research Council and the Department of Health National Institute for Health Research Oxford Biomedical Research Centre; and A.L., T.A., and P.B. are funded by the National Institute for Health Research 0xford Biomedical Centre. We thank Simon Little and Huiling Tan for their help with data collection.

The authors declare no competing financial interests.

Correspondence should be addressed to Peter Brown, Nuffield Department of Clinical Neurology, University of Oxford, Level 6, West Wing, John Radcliffe Hospital, Oxford OX3 9DU, United Kingdom. E-mail: peter.brown@ndcn.ox.ac.uk.

DOI:10.1523/JNEUROSCI.1036-13.2013

Copyright $(2013$ the authors $\quad 0270-6474 / 13 / 3314758-09 \$ 15.00 / 0$
}

ing (Frank, 2006; Cavanagh et al., 2011). In this model, evidence for competing alternatives is integrated until one option reaches the evidence threshold. During conflict, a higher threshold is needed to prevent erroneous responses. One possible mechanism for elevating the evidence threshold is by conflict-triggered movement inhibition. The STN, which receives numerous inputs from cortical areas involved in decision making, is ideally suited for this task. This view has been supported by modeling studies (Frank, 2006; Bogacz and Gurney, 2007), electrophysiological recordings (Brittain et al., 2012; Zaghloul et al., 2012), and behavioral studies (Frank, 2006; Cavanagh et al., 2011).

The mechanism by which the STN might lead to an evidence threshold adjustment, however, is poorly understood. Local field potentials (LFPs) recorded from the STN of PD patients have revealed that situations involving conflict are associated with elevated power levels in the STN theta frequency band $(4-8 \mathrm{~Hz}$; Cavanagh et al., 2011; Fumagalli et al., 2011; Brittain et al., 2012). It has previously been suggested (Cavanagh et al., 2011) that these oscillations may reflect inputs from cortical structures such as the medial prefrontal cortex (mPFC), which project to the STN via the hyperdirect pathway (Smith et al., 1998). The mPFC has been shown to play a role in response inhibition during conflict, most likely through the use of theta $(4-8 \mathrm{~Hz})$ oscillations (Rushworth 
Table 1. Clinical details

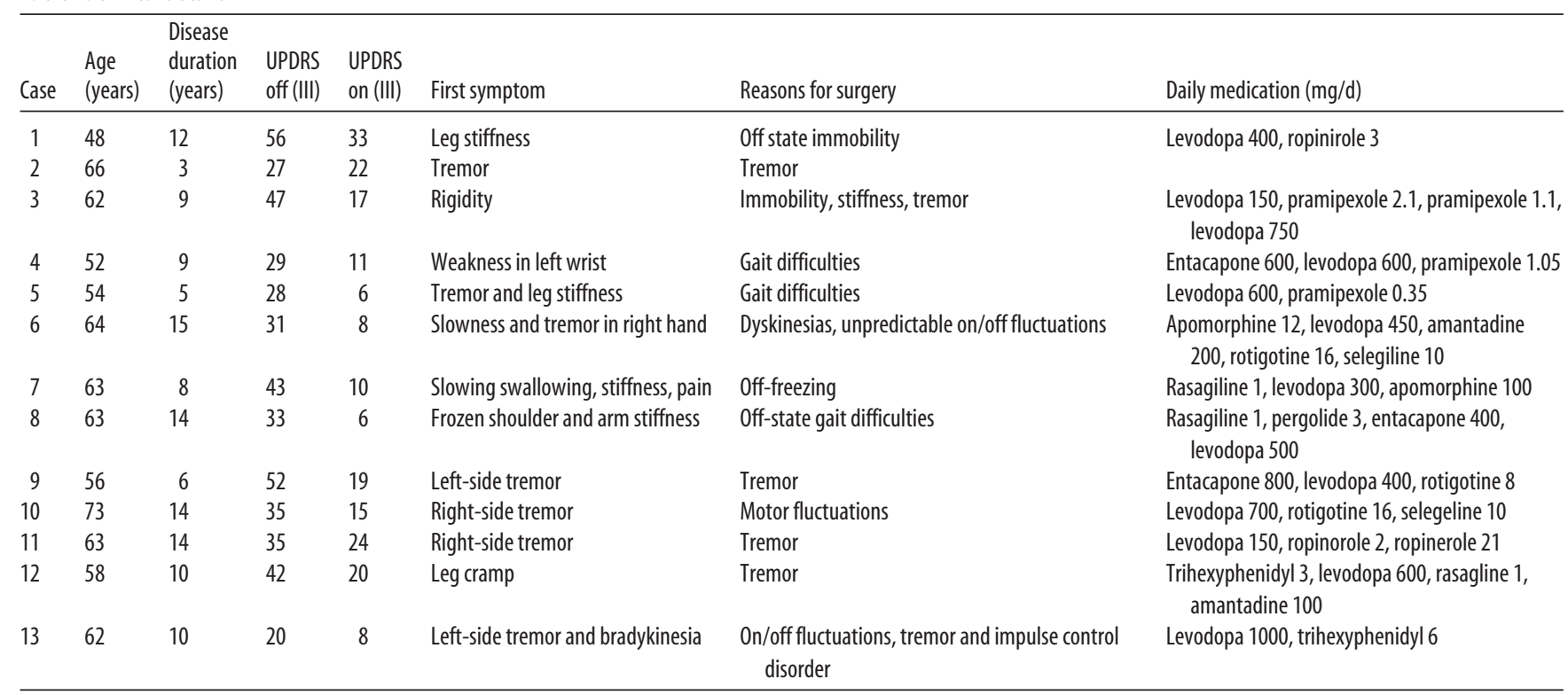

et al., 2004; Cavanagh et al., 2009, 2011; Cohen and Cavanagh, 2011). Furthermore, when Parkinson's disease patients receive DBS to the STN, the relationship between mPFC theta oscillations and reaction time (RT) is disrupted. Though these studies suggest a relationship between theta oscillations in the frontal cortex and the STN, the precise nature of this interaction remains unclear. Is the STN a passive servant to these and other cortical inputs, slowing responses accordingly, or does it actively gate inputs to further fashion behavioral responses?

Here we record LFP activity in the STN in PD patients who have been implanted with DBS electrodes. This was done while patients performed a flanker task, allowing us to define a candidate mechanism by which the STN might gate input during decision making.

\section{Materials and Methods}

Subjects and task. All subjects gave their written informed consent to take part in the study, which was approved by the appropriate local ethics committees. Thirteen subjects (10 males; mean disease duration, 10 years; mean age, 60 years; age range, $48-73$ years) underwent bilateral implantation of DBS electrodes into the STN, as a prelude to highfrequency stimulation for the treatment of advanced PD. Only one patient had been diagnosed as having an impulse control disorder. Techniques to target and implant electrodes in the STN have previously been described (Foltynie and Hariz, 2010). Lead location was confirmed with intraoperative stereotactic MRI at the University College London Hospital and immediate postoperative stereotactic computed tomography at the other centers. Effective stimulation was confirmed intraoperatively in patients operated at the John Radcliffe Hospital and King's College Hospital. The permanent quadripolar electrode used was model 3389 (Medtronic) featuring four platinum-iridium cylindrical surfaces. Electrode extension cables were externalized through the scalp to enable recordings before connection to a subcutaneous DBS pacemaker, implanted in a second operation up to $7 \mathrm{~d}$ later. Clinical details of the patients are available in Table 1 . The mean percentage improvement in the motor section of the Unified Parkinson's Disease Rating Scale (UPDRS) following treatment with levodopa was $59 \pm 5.2 \%(p<0.001$, Wilcoxon signed rank test between ON and OFF levodopa scores) across subjects, indicating good responsiveness to levodopa in our study participants.

Patients performed an arrow version of the flanker task (Eriksen and Eriksen, 1974) while receiving their regular medication 3-6 d after electrode implantation. A schematic of the task is shown in Figure 1A. Each trial began with a black screen containing a white fixation dot in the middle of the screen, which subtended a visual angle of $\approx 1^{\circ}$. Five hundred milliseconds before the arrows were shown, the dot changed from white to gray to prepare the test subject for movement. Either congruent $\left(>>>>>\right.$ ) or incongruent $(<<><<)$ arrows (visual angle $\approx 3^{\circ}$ per arrow) were then briefly shown and replaced with the white fixation dot after $200 \mathrm{~ms}$. The subjects had 2 more seconds in which to respond ( $2.2 \mathrm{~s}$ total possible window for a response) before the fixation dot changed from white to gray again to signal the next trial. Correct responses were indicated by a button press in the hand corresponding to the direction of the middle arrow. The ratio of incongruent trials to congruent trials was 2:1. Subjects underwent two 60-trial blocks. The second block for one subject was discarded due to a sharp drop-off in performance after the first block.

LFP data recording and analysis of power. All signals were sampled at $2048 \mathrm{~Hz}$, bandpass filtered between 0.5 and $500 \mathrm{~Hz}$, and amplified using a TMSi porti and its respective software (TMS International). Monopolar LFP recordings were subsequently converted off-line to a bipolar montage between adjacent contacts (three bipolar channels per side) to limit the effects of volume conduction from distant sources.

Data were analyzed using custom-written Matlab (MathWorks) scripts. For the comparison of correct congruent and incongruent trials, all incorrect responses ( $12 \pm 2 \%$ of all trials) as well as any trials with reaction times $>2 \mathrm{~s}$ (including no response trials) or $<150 \mathrm{~ms}$ were discarded $(<1 \%)$. From the remaining trials, any trial with a response that was $>3$ SDs above the mean latency of the other trials was discarded (mean final trial count, $109 \pm 6.7$ trials per subject). Before further analysis, LFP data were filtered between 1 and $500 \mathrm{~Hz}$ and down-sampled to $1000 \mathrm{~Hz}$. The power and phase of the bipolar LFPs in each trial were calculated using the Morlet wavelet at 8 scales/octave from 2 to $107 \mathrm{~Hz}$. Each trial was analyzed from $0.5 \mathrm{~s}$ before to $2 \mathrm{~s}$ after flanker arrow onset for the cue-aligned analysis, and from $1.5 \mathrm{~s}$ before to $1.5 \mathrm{~s}$ after the response for the response-aligned analysis. A $2 \mathrm{~s}$ buffer on either side was used when calculating the wavelet power to eliminate any edge effects. Any trial with a clear artifact was discarded.

To assess the differences in induced power between congruent and incongruent trials, the following approach was used. First, the mean power in each bipolar recording for each trial type was calculated by averaging the power spectrum across trials. The mean evoked response was also calculated by averaging the raw LFP signal across trials and calculating the power spectrum of the resulting event-related potential. The difference between the mean power spectrum and the evoked power spectrum activity was then calculated to find the induced power spectrum. This method produced a time-frequency image for congruent and incongruent trials for each of the three bipolar contacts on each electrode. Each frequency was then normalized to the overall mean power of 
that frequency across all time points in all trials. Finally, all three bipolar contacts were averaged together, and the difference between the congruent and incongruent trials was calculated. Averaging across all the contact pairs in a given electrode was performed so as to avoid selection bias, although this procedure might serve to underestimate spectral changes in the STN as not all contact pairs were necessarily within or bridging this nucleus. The above procedure was repeated for the electrode of each STN to find the mean difference across subjects. To assess the statistical significance of this difference, the same procedure was repeated 1000 times with the congruent and incongruent labels randomly assigned during each permutation. The $p$ value of each time-frequency pixel was found by comparing the actual mean difference to the distribution of the 1000 permutations. The $p$ values were then corrected for multiple comparisons using exceedence mass testing (Maris and Oostenveld, 2007). Exceedence mass testing involves integrating the excess mass of suprathreshold clusters in the spectrogram and recording the largest per iteration. The top $5 \%$ of this distribution then determined the corrected threshold for image-wise significance. When performing other comparisons (i.e., congruent vs fastincongruent, congruent vs slow-incongruent, fast-incongruent vs slow-incongruent, or incongruent error trials vs correct incongruent trials), the same procedure was repeated using the relevant trial groups. To generate the theta band time-frequency time series, the same procedure was used, but the values across 3-8 $\mathrm{Hz}$ were first averaged before any other calculation.

Intertrial phase locking. To analyze the intertrial phase locking, the LFP signal was bandpass filtered from 4 to $8 \mathrm{~Hz}$, and the Hilbert transform was used to calculate the instantaneous phase of each trial. The phase-locking value (PLV) at each time point was then found by projecting the phase at time $t$ for each trial onto the complex plane and averaging across trials, as follows:

$$
\operatorname{PLV}(t)=\left|\frac{1}{n} \times \sum_{t=1}^{n} e^{i \theta t}\right|
$$

where $n$ is the total number of trials, and $\theta t$ is the phase angle at time $t$. Using this formulation, a PLV $(t)$ value of 0 would mean there is a uniform distribution of phase across trials at time $t$, and a value of 1 would mean that the phase at time $t$ is identical for each trial.

To assess the statistical differences between conditions, the difference between the two PLVs was first calculated for each bipolar signal and averaged across all three bipolar contact pairs of the electrode before being averaged across subjects. The same procedure was then repeated 1000 times with the labels of the two trial groups being randomly assigned at the beginning of each permutation. The $p$ value at each point was calculated using the distribution of the 1000 permuted values and corrected for multiple comparisons at a significance level of 0.05 using exceedence mass testing. To ensure that the results we reported were specific to the $4-8 \mathrm{~Hz}$ band, the same procedure was repeated using the Morlet wavelet for each separate band from 2 to $107 \mathrm{~Hz}$ ( 8 scales/octave).

Electrophysiological relationships with reaction time. To assess the singletrial correlation between reaction time and normalized power changes, the theta power of each trial was averaged across the time period $300 \mathrm{~ms}$ before the response and correlated with the reaction time in that trial. The resulting correlation coefficients (positive and negative coefficients derived using Spearman's correlation) were then averaged across the 26 STN sides and a two-tailed, one-sample $t$ test was performed to determine whether the mean correlation was significantly different from zero across subjects. To determine the presence of a time-on-task confound, the slope of the RT-theta relationship $\left(\theta_{\mathrm{RT}}\right)$ during the congruent trials of each subject was used to adjust the theta power of that subject [adjusted_congruent_theta $=$ congruent_theta $+\theta_{\mathrm{RT}} \times\left(\mathrm{RT}_{\text {slowincongruent }}-\mathrm{RT}\right)_{\text {congruent; }}$; Carp et al., 2010]. As in the correlation analysis, the $300 \mathrm{~ms}$ preceding the response was used to assess significant differences.

The correlation between reaction times and cue-aligned PLV was done by finding the maximum PLV value (calculated across all correct trials regardless of congruency) from stimulus onset until $0.5 \mathrm{~s}$ after the cue for each channel, averaging across all six channels for each subject, and then correlating these values with the average reaction time across subjects using Spearman's correlation. For the response-aligned correlation, the same procedure was performed using the maximum PLV value over a time period from $500 \mathrm{~ms}$ before to $200 \mathrm{~ms}$ after the response.

\section{Results}

\section{Power changes during correct trials}

In accordance with the literature (Eriksen and Eriksen, 1974), the mean reaction time for correct responses was significantly faster for congruent trials than for incongruent trials (Fig. 1B; 484 vs 


\section{Cue Aligned}

A

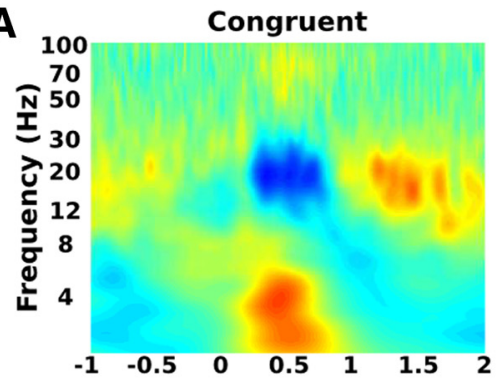

B
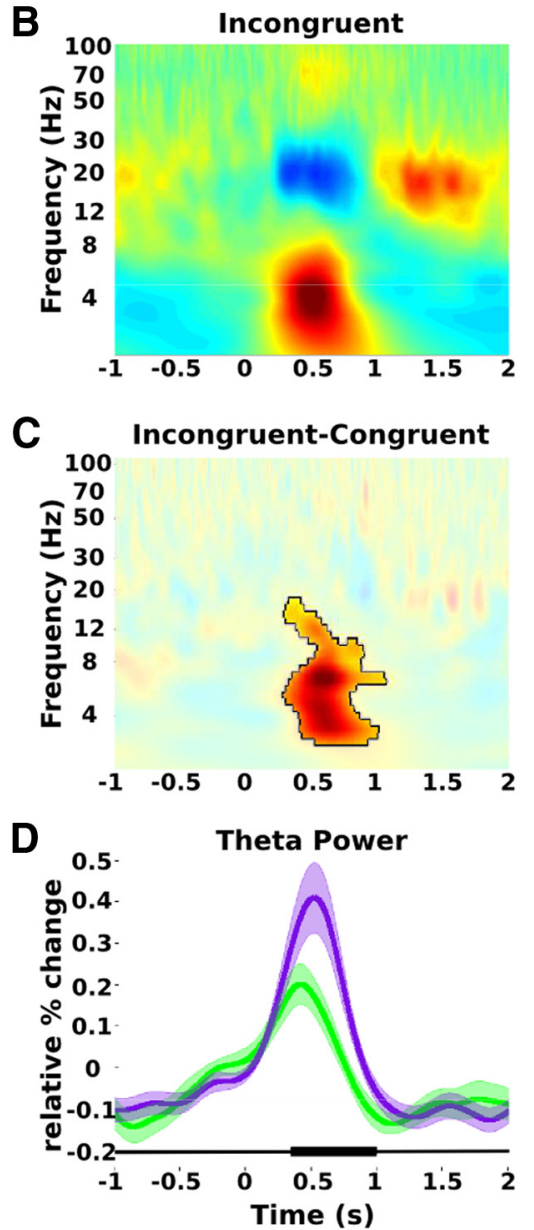

Response Aligned

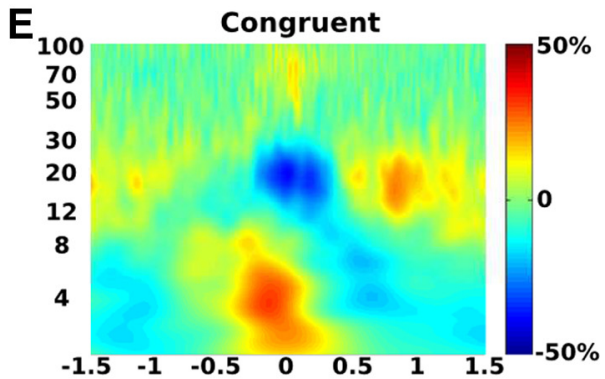

$\mathbf{F}_{100}$
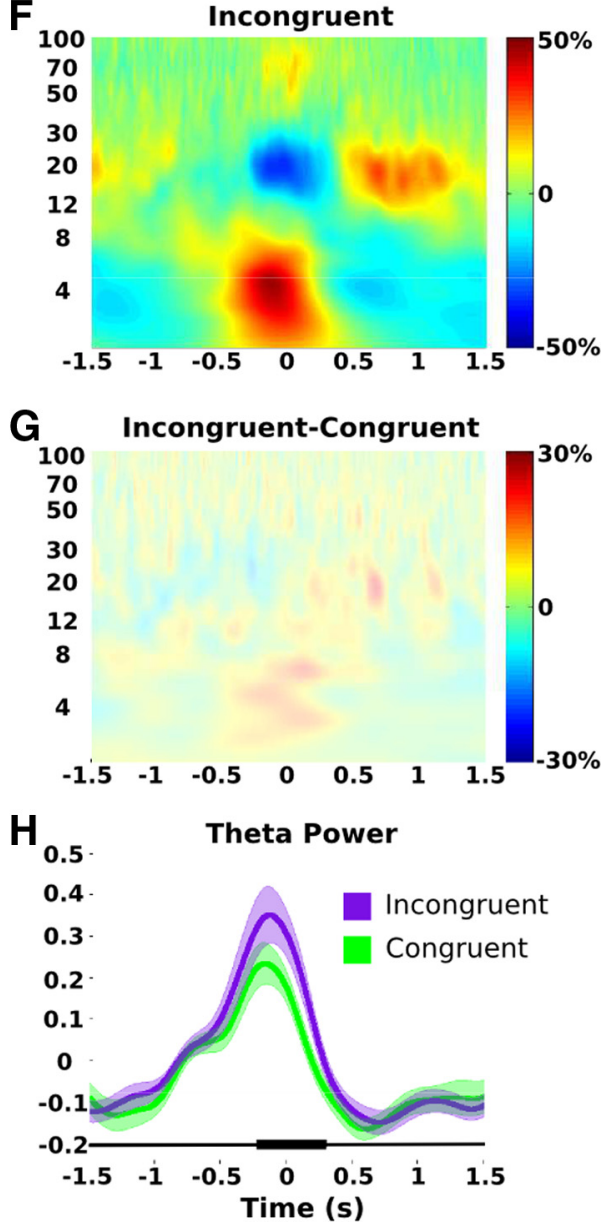

Figure 2. Effects of congruency on LFP across all subjects. $A-D$, Imperative cue-aligned $(t=0)$ averages of induced spectral change. Both congruent $(\boldsymbol{A})$ and incongruent $(\boldsymbol{B})$ trials showed an increase in cue-aligned theta power, a decrease in beta power followed by a postresponse rebound, and an increase in gamma power. $\boldsymbol{C}$, Difference between trial types masked at a 0.05 significance level corrected for multiple comparisons, showing the theta band difference. $\boldsymbol{D}$, Cue-aligned theta $(3-8 \mathrm{~Hz})$ band average time series (mean \pm SEM) for congruent (green) and incongruent (purple) trials. Significant difference between the two conditions is marked by black bar ( $p<0.05$ corrected for multiple comparisons). $\boldsymbol{E}-\boldsymbol{H}$, Same as $\boldsymbol{A}-\boldsymbol{D}$ but aligned to the response. Theta difference is weaker and only significant in the theta band average time series $(\boldsymbol{H})$. Note that here and in ensuing timefrequency plots that frequency is given on a log axis.

$565 \mathrm{~ms} ; p<0.0001$, paired $t$ test). Cue-aligned STN LFP signals in both congruent and incongruent trials showed an increase in theta power, a decrease in beta power followed by a postresponse beta rebound, and an increase in gamma power (Fig. 2A,B). Power differences between congruent and incongruent trials revealed higher preresponse, conflict-related activity focused in the theta $(3-8 \mathrm{~Hz}$ ) band (Fig. $2 C, D ; p<0.05)$. The higher preresponse theta power was also evident in the response-aligned data (Fig. $2 H ; p<0.05$ ). The effect, however, was weaker than with cue aligning, as evidenced by the lack of significance when the power difference was corrected for multiple comparisons across the entire spectrogram (Fig. 2G).

We used a 2:1 ratio of incongruent to congruent trials, and therefore were able to divide the trials into three groups containing an approximately equal number of trials: congruent trials, the fastest half of the incongruent trials, and the slowest half of the incongruent trials (Fig. 1B,C). A histogram of normalized reaction times for the incongruent trials provided further support for this procedure by suggesting a bimodal distribution of incongruent reaction times (Fig. 1C). Figure 3 contrasts the STN LFP time-frequency profiles between trial types. When the congruent trials were compared with the fast-incongruent trials there was no significant difference in reaction time (Fig. $1 B$ ) or induced power (Fig. $3 A, D, E, H)$. On the other hand, when the slow-incongruent trials were compared with the congruent trials, slower reaction times were observed (Fig. 1B), and induced theta power was higher in the slowincongruent trials (Fig. $3 B, D, F, H$ ). The theta power differences were also observed when the slow-incongruent trials were compared with the fast-incongruent trials (Fig. 3C, D, G,H). Aligning the data to the response revealed that the higher theta power observed during the slowincongruent trials was mostly confined to the preresponse period (Fig. $3 F-H$ ).

Figure 3 suggests that the differences between congruent and incongruent trials shown in Figure 2 were due to the slowincongruent trials. To test whether there was a relationship between theta power and reaction time at the single-trial level, we correlated the reaction time of each trial to the mean theta power in the $300 \mathrm{~ms}$ preceding the response. This revealed a significant, albeit low, positive correlation for both the congruent (mean $r=0.17 \pm .03, p<0.001$, single-sample $t$ test) and the incongruent trials (mean $r=0.08 \pm 0.04, p<0.05$, single-sample $t$ test). The strength of the correlation was not significantly different between the congruent and the incongruent trials $(p=0.07$, paired $t$ test $)$.

Though the correlations we observed are small, they raise the possibility of a time-on-task effect (Carp et al., 2010). The interpretation of this effect is a subject of debate (Nachev, 2011; Yeung et al., 2011; Cohen and Nigbur, 2013; Scherbaum and Dshemuchadse, 2013), but at the very least it serves to highlight a potential ambiguity in interpretation. Does this correlation with reaction time come about because reaction time is an index of conflict (Yeung et al., 2004) and the primary link is between high theta power and conflict, or is high theta power related to other undefined processes that determine reaction time? Indeed, when the theta 
power observed in the congruent trials was adjusted to account for the longer reaction times the slow-incongruent trials were no longer significantly different (incongruent vs adjusted congruent, $0.41 \pm 0.09$ vs $0.42 \pm 0.10$, normalized theta power change \pm SEM; $p>0.05$, paired $t$ test; Carp et al., 2010; see Materials and Methods). Unfortunately, there were too few congruent trials with long enough reaction times (mean, 7 trials; range, 2-12 trials) to be directly compared with slow-incongruent trials.

Theta phase intertrial phase locking during correct trials

Several flanker paradigm studies have demonstrated that cortical theta frequency oscillations demonstrate phase reorganization during the task (Cavanagh et al., 2009; Cohen and Cavanagh, 2011). Accordingly, we tested whether a similar phenomenon occurred in the STN. Figure $4, A$ and $B$, illustrates the average cuealigned wavelet PLV (see Materials and Methods) for the fast-incongruent (Fig. $4 A$ ) and the slow-incongruent trials (Fig. $4 B$ ). Subtracting the cue-locked PLV matrices across subjects revealed a significantly lower intertrial theta phase locking during the slow-incongruent trials (Fig. $4 C$ ). To explore this phenomenon further, we bandpass filtered the data between 4 and $8 \mathrm{~Hz}$ and compared the PLV across the three conditions (congruent, fastincongruent, and slow-incongruent) using the Hilbert transform (Fig. 4D). All trial types demonstrated a small PLV increase following the warning cue $(t=$ $-500 \mathrm{~ms}$ ), and a second, larger increase upon presentation of the arrows, peaking 140 to $200 \mathrm{~ms}$ after the arrows were shown. Though we did not see any systematic differences in absolute phase between trial types $\left(11 \pm 7^{\circ}\right.$, mean phase difference \pm SEM; $p>0.05$, Wilcoxon signed rank test), the slow-incongruent trials demonstrated a significantly lower PLV upon arrow presentation than both the congruent trials and the fast-incongruent trials (Fig. $4 A-D$ ). These differences were significant when the point-wise PLV values were compared (Fig. 4D) and when the maximum PLV value between 0 and $500 \mathrm{~ms}$ was compared ( $p<0.01$ for slow vs fast-incongruent and slowincongruent versus congruent; $p>0.2$ for fast-incongruent vs congruent; paired $t$ tests). This suggests that during the slowincongruent trials the stimulus triggered decrease in theta phase variability was impaired.

Several lines of evidence strongly suggest that the impairment of phase realignment in the slow-incongruent trials was not due to their longer and potentially more variable reaction times. First, the congruent trials showed a higher peak PLV despite having a
Cue Aligned

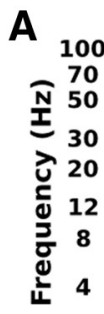

B


Response Aligned

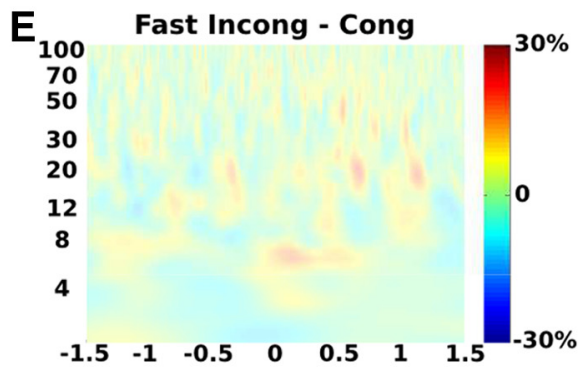

$\mathbf{F}$

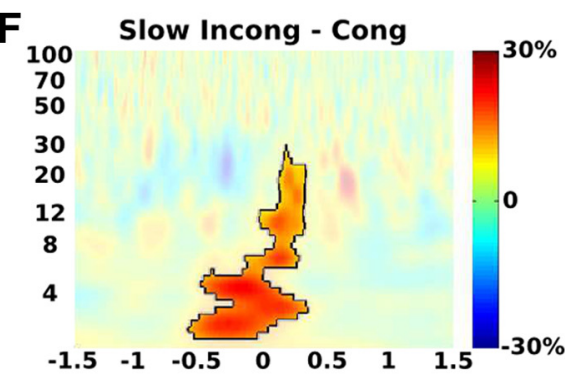

G
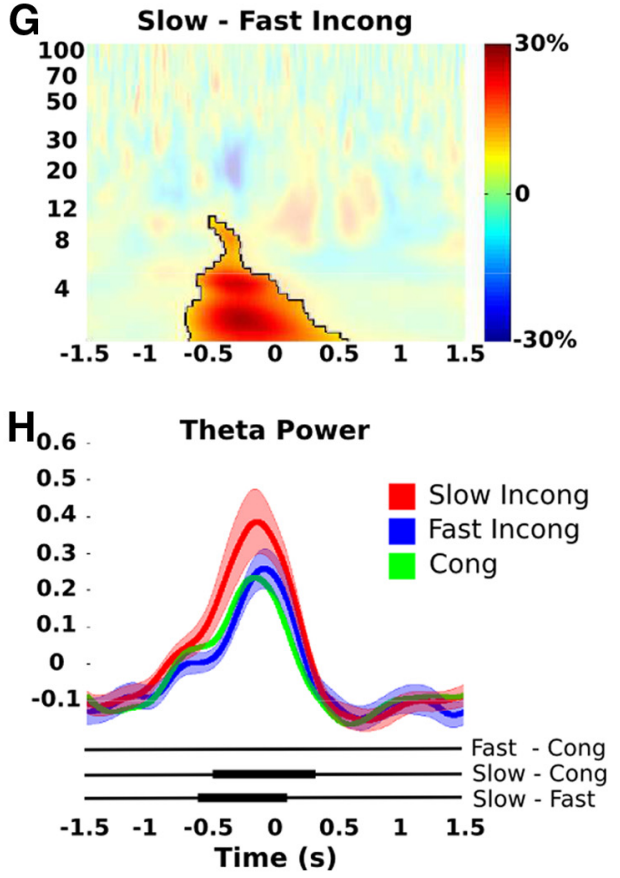

Figure 3. Differences between slow and fast trials following incongruent cues across all subjects. $\boldsymbol{A}-\boldsymbol{D}$, Imperative cue-aligned $(t=0)$ averages of induced spectral change. $\boldsymbol{A}$, No power differences between the fast-incongruent trials and congruent trials (masked at $p<0.05$ significance level after correcting for multiple comparisons). B, C, Slow-incongruent trials showed higher cue-aligned theta power than congruent trials $(\boldsymbol{B})$ and fast-incongruent trials $(\boldsymbol{C}$ ). $\boldsymbol{D}$, Theta $(3-8 \mathrm{~Hz})$ band average time series for slow-incongruent (red), fast-incongruent (blue), and congruent (green) trials. Note that mean \pm SEM values are shown except for congruent trials (where \pm SEM values were shown in Fig. 2). Significant difference between trial types is marked by horizontal bars ( $p<0.05$, corrected for multiple comparisons). $\boldsymbol{E}-\boldsymbol{H}$, Same as $\boldsymbol{A}-\boldsymbol{D}$ but aligned to the response. Fast-incongruent trials showed no significant difference from the congruent. Preresponse theta power was higher in slow-incongruent trials. Cong, Congruent. much more variable reaction time than the slow-incongruent trials (mean \pm SEM, $81 \pm 6$ vs $57 \pm 6$; $p<1 \times 10^{-7}$; for a comparison of the distributions, see Fig. 1). Second, when the congruent trials were median split and analyzed in the same way as the median split incongruent trials, slow and fast congruent trials showed no difference in their cue-locked theta PLV increase (neither point-wise comparisons nor comparisons of the maximum PLV value showed any differences; data not shown). This suggests that the difference between the fast- and slow- 

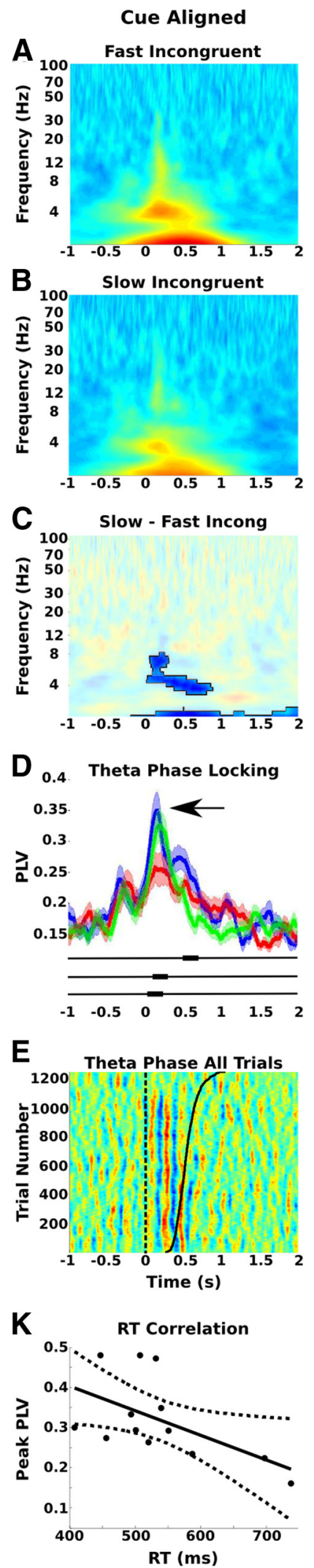
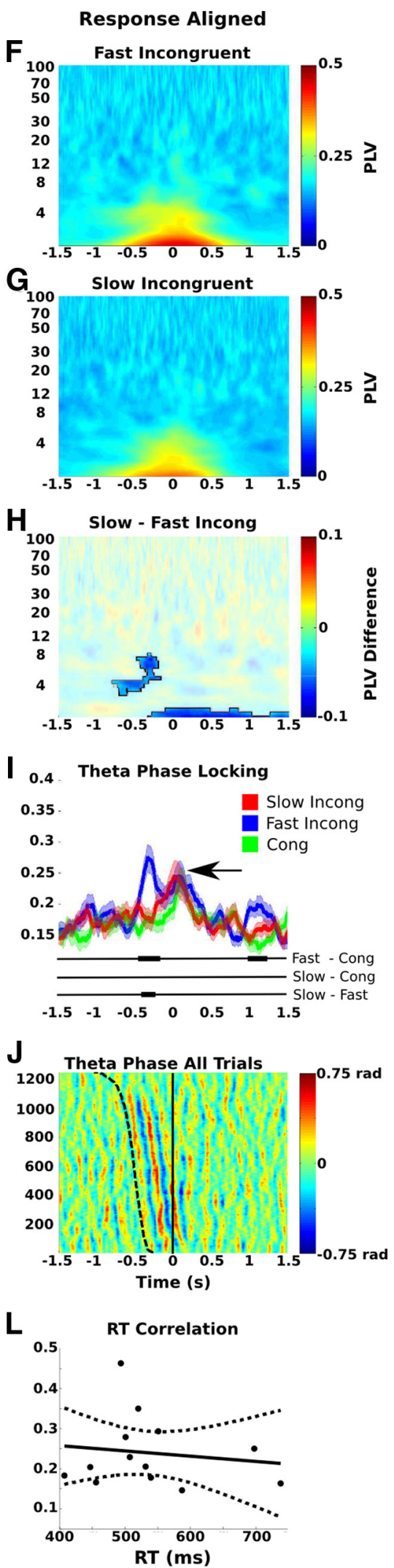

Figure 4. Cue-locked theta phase realignment is disrupted in slow-incongruent trials. $\boldsymbol{A}-\boldsymbol{E}$, Imperative cue-aligned $(t=0)$ averages of phase locking across trials. $A$, Mean wavelet PLV for all fast-incongruent trials averaged across all 26 STNs. $B$, Same as $\boldsymbol{A}$ for slow-incongruent trials. $\boldsymbol{C}$, There is a significant reduction in cue-locked theta PLV when the slow-incongruent trials are compared with fast-incongruent trials (masked at $p<0.05$ significance level after correcting for multiple comparisons). $\boldsymbol{D}$, Theta band filtered Hilbert PLV also showed impaired cue-locked phase alignment in the slow-incongruent trials. Slow-incongruent (red), fast-incongruent (blue), and congruent (green) mean PLV time series are shown \pm SEM. Significant differences are denoted incongruent trials was not simply due to reaction time differences. Finally, the phase alignment was locked to the cue and peaked $\sim 300$ ms before the average response, which occurred between 485 and $640 \mathrm{~ms}$ after the cue, depending on trial congruency (Fig. 4D). This cue locking was particularly well seen when the theta phase from all correct trials recorded in all patients was aligned to the cue (Fig. 4E; sorted by reaction time, the channel with the clearest cue-locked phase alignment was used for each patient). These showed a prominent increase in phase synchrony locked to the presentation of the arrows at time $t=0$.

To verify that the phase alignment just after the imperative cue (arrows) was indeed locked to the cue rather than the response, we repeated the same analysis with the data locked to the response. This revealed that there was another, weaker period of phase realignment that occurred when subjects made their response. The response-locked increase in phase locking peaked between 20 and $100 \mathrm{~ms}$ after the response was made, which was $\sim 400 \mathrm{~ms}$ after the peak in the cue-aligned PLV increase (Fig. $4 F, G, I$ ). This suggests that the cue-aligned PLV increase and the response-aligned PLV increase were two separate events. This is supported by the fact that two peaks can be seen in the response-aligned fast-incongruent trials (Fig. 4I). Both peaks are most likely visible only in the fast-incongruent trials because these trials had the least variable reaction times (fast-incongruent vs congruent mean SD \pm SEM, $39 \pm 2$ vs $81 \pm 6 \mathrm{~ms}, p<$ $1 \times 10^{-8}$; fast-incongruent vs slow-

\footnotetext{
by horizontal bars. $\boldsymbol{E}$, Theta bandpass-filtered Hilbert phase for all trials in all subjects sorted by reaction time (smoothed across each 100 trials and $40 \mathrm{~ms}$ ). Prominent phase alignment is stimulus locked and independent of reaction time. Dotted black track indicates stimulus onset, and solid black trace indicates trial reaction time. $\boldsymbol{F}-\boldsymbol{J}$, Same as $\boldsymbol{A}-\boldsymbol{D}$ but aligned to response. Comparison of $\boldsymbol{D}$ and $\boldsymbol{I}$ suggests that there are two phase alignment periods: just after but time locked to stimulus onset ( $\boldsymbol{D}$, arrow pointing to large peak) and periresponse $(\boldsymbol{I}$, arrow pointing to second peak centered on response at $t=0$ ). Both peaks can be seen in the response-aligned Hilbert PLV of the fast-incongruent trials, which showed the least variable reaction time $(\boldsymbol{I})$. Cue-locked phase alignment $(\boldsymbol{D})$ is greater than response-aligned phase alignment $(I)$ for all trial types except slow-incongruent trials, which show impaired cuelocked phase alignment. $\boldsymbol{K}$, Cue-locked phase alignment (calculated across all correct congruent and incongruent trials) correlated with subject reaction time $(r=-0.58, p<0.05)$. $L$, Response-locked phase alignment did not correlate with subject reaction time $(r=-0.11)$. Correlations show the results of linear regression and corresponding 95\% confidence limits. Cong, Congruent.
} 
incongruent mean SD \pm SEM, $39 \pm 2$ vs $57 \pm 6 \mathrm{~ms}, p<0.01)$. In fact, the responsetriggered increase in PLV for the fastincongruent trials may also potentially be responsible for the second, small relative increase in the cue-aligned fast-incongruent trial data in Figure $4 D$ (blue trace at approximately $t=500 \mathrm{~ms}$ ), where it is superimposed on the falling end of the earlier cue-locked PLV peak.

Further evidence that the cue-locked increase in theta PLV is not due to "smearing" from a response-locked theta PLV increase stems from the fact that the former is larger than the latter (Fig. $4 D, I$, compare black arrows). When the theta phase from all correct trials recorded in all patients was aligned to the response (Fig. 4J; sorted by reaction time), a diagonal trend parallel to the onset of each trial (Fig. 4J, dotted black trace) could be seen. This suggests that the dominant theta phase alignment effect was locked to the cue and not to the response. In line with this, the cue-aligned peak was significantly higher than the response-aligned peak when the PLV was calculated across all trials (regardless of arrow congruency), and across all congruent trials, incongruent trials, and fast-incongruent trials $(p<0.05$ for all comparisons, paired $t$ test). The only trials that did not show a significant difference in the peak PLV between the cue and the response-aligned data were the slow-incongruent trials. This was due to the disruption of the cue-aligned phase locking during the slow-incongruent trials, as mentioned above.

Unlike the cue-aligned increase in phase locking, the maximum value of the response-aligned increase (between -200 and $200 \mathrm{~ms})$ showed no difference between trial types $(p>0.05$, paired $t$ test). This, together with the timing of the two peaks, suggests that only the cue-aligned PLV increase may play a functional role in determining reaction time. This is supported by the finding that when the PLV was computed across all correct trials (regardless of congruency), the maximum cue-aligned PLV correlated with reaction time across subjects (Fig. $4 K ; r=-0.58, p<$ $0.05)$. When the data were aligned to the response, on the other hand, there was no significant correlation between PLV and reaction time (Fig. $4 L ; r=-0.11, p>0.05$ ).

\section{Power changes in error trials}

Finally, we contrasted the spectral changes between the correct incongruent and the incorrect incongruent trials $(16.4 \pm 2.8 \%$; only $4.9 \pm 1.6 \%$ of congruent trials were incorrect- too few to perform such an analysis). Incorrect incongruent trials had faster reaction times than correct slow-incongruent trials, but not correct fast-incongruent trials (Fig. 5A). As with the correct trials, the incorrect trials demonstrated an increase in theta power, a decrease in beta power, and an increase in gamma power (Fig. $5 B)$. When the incorrect-incongruent trials were compared with the correct-incongruent trials, a higher delta power $(2-4 \mathrm{~Hz})$ was seen in both the cue and response-aligned data (Fig. $5 B-E$ ), and a significant increase in $10-20 \mathrm{~Hz}$ power after the response in response-aligned data (Fig. $5 D, E$ ).

\section{Spatial focality of recordings}

Here we present the results for the average across all contract pairs of the deep brain stimulation electrode targeting the STN on different sides across patients. LFP recordings from the subthalamic region were highly focal, as indexed by steep percentage drops in power when comparing the contact pair with the highest absolute power to the mean power recorded by the two remaining contact pairs on each electrode. Relative to the best contact pair, the relative mean theta power for the two remaining channels dropped to $48.4 \pm 4.1$ (mean \pm SEM). When the congruent versus incongruent trial comparisons were repeated using only the channel with the highest beta or theta power, the same relative increase in theta power and decrease in theta PLV were observed during the slow-incongruent trials (data not shown). Though the variability of contact locations across each STN did not allow us to find any significant topographical segregation of the reported effects on theta power and phase, we found that, on average, the contact with the highest beta power was less deep than the contact with the highest theta power by 0.31 times the intercontact distance (or $0.62 \mathrm{~mm}$ if we take this distance as that between the center of each contact). Although this difference is compatible with the hypothesis that theta activity is more focused in associative and limbic STN and beta activity is more focused in dorso- 
lateral motor STN, the difference was not statistically significant $(p=0.151$, Wilcoxon rank sum test).

\section{Discussion}

Our results suggest that STN LFP activity tends to present one of three spectral variants depending on flanker trial type. Correct congruent and fast-incongruent trials demonstrated similar theta phase realignment when the arrows were shown, followed by a comparable theta power increase. The similarity in LFP response between the two trial types was associated with similar reaction times and may imply that subjects were able to appropriately ignore the distracting flankers during correct fast-incongruent trials. The slowest correct incongruent trials, where we presume that the distracting arrows were attended to, resulting in conflict, were associated with a disruption of cue-aligned theta phase reset, a higher theta power increase, and longer reaction times. This observation is consistent with other studies that have also reported higher STN theta power during high-conflict trials (Cavanagh et al., 2011; Fumagalli et al., 2011; Brittain et al., 2012). The final variant of STN spectral activity occurred during incorrect responses, which showed higher delta $(2-4 \mathrm{~Hz})$ and alpha/low beta $(10-20 \mathrm{~Hz})$ power, but only during or after the response. Error-related delta power increases have previously been observed over mPFC (Yordanova et al., 2004; Cavanagh et al., 2012).

Before further interpretation, we should acknowledge three possible limitations of the present study. First, participants were necessarily PD patients who had undergone surgery, so inferences with regard to normal functioning must be circumspect (Williams et al., 2002). That said, recordings were made while patients were receiving levodopa, while their clinical state was as near to normal as possible. Second, even depth recordings of LFPs can be subject to volume conduction of activities from cerebral cortex. To mitigate this, we recorded in a bipolar configuration from the contacts of the DBS electrode and demonstrated a steep gradient in LFP power between contact pairs, consistent with a local generator (Kühn et al., 2004, 2006). Last, the correlations between spectral features and reaction times across trials were relatively weak, albeit significant. There are reasons why this might be, including the multiplicity of variables contributing to reaction time and the postoperative stun effect that may serve to attenuate local power reactivity (Chen et al., 2006). These factors may also explain why, unlike the correlations between theta power and reaction time that have been observed over the $\mathrm{mPFC}$ during the flanker task (Cohen and Cavanagh, 2011), the correlations we observed were not significantly stronger in the incongruent condition.

With the above caveats in mind, our findings provide evidence that the STN is involved in the processing of congruent and incongruent responses, and may impact successful response selection. As such, our results add to a growing body of evidence supporting model-based hypotheses that the STN plays a crucial role in decision making during conflict (Frank, 2006; Bogacz and Gurney, 2007). Not only does disruption of the STN lead to impulsivity (Baunez and Robbins, 1997; Frank et al., 2007; Ray et al., 2009; Cavanagh et al., 2011; Coulthard et al., 2012), it has also, as here, been shown to exhibit conflict-related electrophysiological changes (Cavanagh et al., 2011; Fumagalli et al., 2011; Brittain et al., 2012; Zaghloul et al., 2012).

How might different STN spectral features help determine response timing under conditions of conflict? We have identified two temporally separate events in the STN that are reflected in the LFP. The first is a theta band phase reorganization that follows the imperative cue at short latency, and is bigger in congruent and fast- incongruent trials than in slow-incongruent trials. This feature is phase locked to the imperative cue and not the response, as demonstrated by the twofold decrease in average magnitude when trials are realigned to response as opposed to stimulus onset. These observations raise the possibility that the theta band phase reorganization reflects an early subcortical response related to the cue that nevertheless then impacts the speed with which the relevant behavior is processed. The latter is supported by the negative correlation between cue-locked phase reorganization and reaction time. When flanking arrows are attended to, the competing activation due to the conflicting arrows may serve to attenuate theta band phase reorganization and thus prolong reaction time.

The second event is an increase in STN theta band power. It follows the cue-locked theta band phase reorganization and peaks just before the response. Unlike the theta phase reorganization, its magnitude is not significantly reduced when trials are realigned to response onset, as opposed to cue onset. Together, these observations suggest that theta power is at least partially linked to the response itself, possibly helping to determine its timing. There are two principal ways in which an event might determine the timing of a behavioral response. First, the event might trigger it (either directly or through removal of inhibition). In this case, the event should begin at the same time relative to the response. The response aligning of theta power, however, suggests that theta power in the slow-incongruent trials begins increasing before that in fast-incongruent and congruent trials. Thus, the onset of the theta power increase per se cannot trigger the response, but the response might be triggered when it reaches a certain threshold amplitude. The fact that theta power reaches a higher level before the response in slow-incongruent trials would then imply that the theta power increase has to go on for longer so as to reach a higher threshold in this trial type. This interpretation is supported by the similar slopes of the initial theta power increases, most clearly seen in the cue-aligned trials and also by the positive correlation between theta power preresponse and reaction time. It is for this reason that the difference in theta power between trial types may have been lost when reaction time was controlled for (Carp et al., 2010).

It is important to stress that all correct responses had both events, an early theta band phase reorganization and a later theta power increase, and that these only varied in their relative dominance between trial types. Could responses rely on both processes? Ordinarily during congruent trials, arrows may elicit a prominent theta band phase reorganization. After this, the subsequent theta power increase may only have to reach a low threshold to trigger a response, resulting in fast reaction times. In fast-incongruent trials, the flanking arrows are not attended to and the LFP pattern does not change. However, in slowincongruent trials, failure to ignore the flanking arrows diminishes the phase reorganization, and without this step the subsequent theta power increase has to reach a higher level before a response can be triggered. This takes longer. In effect, the weaker initial phase reorganization might set the subsequent theta power threshold higher. This interpretation is consistent with current models of response selection under conditions of conflict, where the STN is afforded a critical role in adjusting the response threshold (Frank, 2006; Cavanagh et al., 2011). Though it is tempting to associate the theta changes seen here with this process, it is also important to note that others have postulated an inhibitory role for conflict-related increases in STN theta power (Cavanagh et al., 2011).

Lacking in our above hypothetical scheme is a means by which response-locked theta band phase reorganization could modu- 
late subsequent processing. EEG studies, however, may provide some clues. These reveal theta phase reorganization in the $\mathrm{mPFC}$ in several different paradigms (Cohen et al., 2008; Cavanagh et al., 2009, 2010, 2012; Cohen and Cavanagh, 2011). Recently, Cohen and Cavanagh (2011) have shown that mPFC theta phase in the flanker task correlates with reaction time up to $500 \mathrm{~ms}$ before response occurs. In other words, some mPFC theta phase values predicted long reaction times, and others predicted short reaction times. Here, we have demonstrated that the STN shows theta phase realignment when an imperative cue is given, and that the degree of alignment across trials negatively correlates with reaction time across subjects. One-way theta phase resetting could affect function is through promoting or disrupting optimum phase alignment with relevant afferent and efferent targets (Fries, 2005). For example, studies have shown that the degree of theta phase coupling between the MPFC and inferior frontal gyrus following errors correlates with posterror slowing on the following trial (Cavanagh et al., 2009; Cohen and Cavanagh, 2011). In the context of communication through coherence (Fries, 2005), the disruption of STN phase alignment during slow-incongruent trials may reveal a conflict-induced increased net deviation from the ideal phase for rapid response execution.

Our formulation acknowledges both the sequential timing of STN theta phase realignments and power increases as well as the contrasting signs of their respective correlations with reaction time. However, there is still much that remains to be corroborated and clarified. For example, it remains to be determined whether the conflict-related STN theta activity is driven via mPFC (Cavanagh et al., 2011) or via cortical-striatal inputs. Nor need the relative disorganization of theta phase be the only STN process resulting in delayed response selection (Brittain et al., 2012). Nevertheless, the current findings suggest how the synchronized activity of neuronal populations in the STN may help operationalize the hold-your-horses function of this nucleus.

\section{References}

Albin RL, Young AB, Penney JB (1989) The functional anatomy of basal ganglia disorders. Trends Neurosci 12:366-375. CrossRef Medline

Baunez C, Robbins TW (1997) Bilateral lesions of the subthalamic nucleus induce multiple deficits in an attentional task in rats. Eur J Neurosci 9:2086-2099. CrossRef Medline

Bogacz R, Gurney K (2007) The basal ganglia and cortex implement optimal decision making between alternative actions. Neural Comput 19:442-477. CrossRef Medline

Brittain JS, Watkins KE, Joundi RA, Ray NJ, Holland P, Green AL, Aziz TZ, Jenkinson N (2012) A role for the subthalamic nucleus in response inhibition during conflict. J Neurosci 32:13396-13401. CrossRef Medline

Carp J, Kim K, Taylor SF, Fitzgerald KD, Weissman DH (2010) Conditional differences in mean reaction time explain effects of response congruency, but not accuracy, on posterior medial frontal cortex activity. Front Hum Neurosci 4:231. CrossRef Medline

Cavanagh JF, Cohen MX, Allen JJ (2009) Prelude to and resolution of an error: EEG phase synchrony reveals cognitive control dynamics during action monitoring. J Neurosci 29:98-105. CrossRef Medline

Cavanagh JF, Frank MJ, Klein TJ, Allen JJ (2010) Frontal theta links prediction errors to behavioral adaptation in reinforcement learning. Neuroimage 49:3198-3209. CrossRef Medline

Cavanagh JF, Wiecki TV, Cohen MX, Figueroa CM, Samanta J, Sherman SJ, Frank MJ (2011) Subthalamic nucleus stimulation reverses mediofrontal influence over decision threshold. Nat Neurosci 14:1462-1467. CrossRef Medline

Cavanagh JF, Zambrano-Vazquez L, Allen JJ (2012) Theta lingua franca: a common mid-frontal substrate for action monitoring processes. Psychophysiology 49:220-238. CrossRef Medline

Chen CC, Pogosyan A, Zrinzo LU, Tisch S, Limousin P, Ashkan K, Yousry T, Hariz MI, Brown P (2006) Intra-operative recordings of local field po- tentials can help localize the subthalamic nucleus in Parkinson's disease surgery. Exp Neurol 198:214-221. CrossRef Medline

Cohen MX, Cavanagh JF (2011) Single-trial regression elucidates the role of prefrontal theta oscillations in response conflict. Front Psychol 2:30. CrossRef Medline

Cohen MX, Nigbur R (2013) Reply to "Higher response time increases theta energy, conflict increases response time.” Clin Neurophysiol 124:1479-1481. CrossRef

Cohen MX, Ridderinkhof KR, Haupt S, Elger CE, Fell J (2008) Medial frontal cortex and response conflict: evidence from human intracranial EEG and medial frontal cortex lesion. Brain Res 1238:127-142. CrossRef Medline

Coulthard EJ, Bogacz R, Javed S, Mooney LK, Murphy G, Keeley S, Whone AL (2012) Distinct roles of dopamine and subthalamic nucleus in learning and probabilistic decision making. Brain 135:3721-3734. CrossRef Medline

DeLong MR (1990) Primate models of movement disorders of basal ganglia origin. Trends Neurosci 13:281-285. CrossRef Medline

Eriksen BA, Eriksen CW (1974) Effects of noise letters upon the identification of a target letter in a nonsearch task. Percept Psychophys 16:143-149. CrossRef

Foltynie T, Hariz MI (2010) Surgical management of Parkinson's disease. Expert Rev Neurother 10:903-914. CrossRef Medline

Frank MJ (2006) Hold your horses: a dynamic computational role for the subthalamic nucleus in decision making. Neural Netw 19:1120-1136. CrossRef Medline

Frank MJ, Samanta J, Moustafa AA, Sherman SJ (2007) Hold your horses: impulsivity, deep brain stimulation, and medication in parkinsonism. Science 318:1309-1312. CrossRef Medline

Fries P (2005) A mechanism for cognitive dynamics: neuronal communication through neuronal coherence. Trends Cogn Sci 9:474-480. CrossRef Medline

Fumagalli M, Giannicola G, Rosa M, Marceglia S, Lucchiari C, Mrakic-Sposta S, Servello D, Pacchetti C, Porta M, Sassi M, Zangaglia R, Franzini A, Albanese A, Romito L, Piacentini S, Zago S, Pravettoni G, Barbieri S, Priori A (2011) Conflict-dependent dynamic of subthalamic nucleus oscillations during moral decisions. Soc Neurosci 6:243-256. CrossRef Medline

Kühn AA, Williams D, Kupsch A, Limousin P, Hariz M, Schneider GH, Yarrow K, Brown P (2004) Event-related beta desynchronization in human subthalamic nucleus correlates with motor performance. Brain 127: 735-746. CrossRef Medline

Kühn AA, Doyle L, Pogosyan A, Yarrow K, Kupsch A, Schneider GH, Hariz MI, Trottenberg T, Brown P (2006) Modulation of beta oscillations in the subthalamic area during motor imagery in Parkinson's disease. Brain 129:695-706. CrossRef Medline

Maris E, Oostenveld R (2007) Nonparametric statistical testing of EEG- and MEG-data. J Neurosci Methods 164:177-190. CrossRef Medline

Nachev P (2011) The blind executive. Neuroimage 57:312-313. CrossRef Medline

Ray NJ, Jenkinson N, Brittain J, Holland P, Joint C, Nandi D, Bain PG, Yousif N, Green A, Stein JS, Aziz TZ (2009) The role of the subthalamic nucleus in response inhibition: evidence from deep brain stimulation for Parkinson's disease. Neuropsychologia 47:2828-2834. CrossRef Medline

Rushworth MF, Walton ME, Kennerley SW, Bannerman DM (2004) Action sets and decisions in the medial frontal cortex. Trends Cogn Sci 8:410-417. CrossRef Medline

Scherbaum S, Dshemuchadse M (2013) Higher response time increases theta energy, conflict increases response time. Clin Neurophysiol 124: 1477-1479. CrossRef Medline

Smith Y, Bevan MD, Shink E, Bolam JP (1998) Microcircuitry of the direct and indirect pathways of the basal ganglia. Neuroscience 86:353-387. CrossRef Medline

Williams D, Tijssen M, Van Bruggen G, Bosch A, Insola A, Di Lazzaro V, Mazzone P, Oliviero A, Quartarone A, Speelman H, Brown P (2002) Dopamine-dependent changes in the functional connectivity between basal ganglia and cerebral cortex in humans. Brain 125:1558-1569. CrossRef Medline

Yeung N, Botvinick MM, Cohen JD (2004) The neural basis of error detection: conflict monitoring and the error-related negativity. Psychol Rev 111:931-959. CrossRef Medline

Yeung N, Cohen JD, Botvinick MM (2011) Errors of interpretation and 
modeling: a reply to Grinband et al. Neuroimage 57:316-319. CrossRef Medline

Yordanova J, Falkenstein M, Hohnsbein J, Kolev V (2004) Parallel systems of error processing in the brain. Neuroimage 22:590-602. CrossRef Medline
Zaghloul KA, Weidemann CT, Lega BC, Jaggi JL, Baltuch GH, Kahana MJ (2012) Neuronal activity in the human subthalamic nucleus encodes decision conflict during action selection. J Neurosci 32:2453-2460. CrossRef Medline 